\title{
Temperaturkalibrator mit schneller Temperierung des Prüflings
}

\author{
Dr. René Friedrichs, Dr. Eike Christian Weiß \\ SIKA Dr. Siebert \& Kühn GmbH \& Co. KG, Struthweg 7 - 9, 34260 Kaufungen (Germany)
}

\section{Zusammenfassung}

Wir haben ein Regelverfahren entwickelt, mit dem sich die Zeit, innerhalb der der Prüfling das thermische Gleichgewicht mit der Temperiereinrichtung erreicht, deutlich reduzieren lässt. Dazu wird nicht direkt auf die Anzeige des Referenzsensors des Temperaturkalibrators sondern auf die daraus mittels eines thermischen Modells berechnete Temperatur des Prüflings geregelt. Mit diesem Ansatz lässt sich die Wartezeit bis zum eigentlichen Kalibriervorgang je nach Wärmekapazität der Temperiereinrichtung bzw. des Prüflings merklich reduzieren. Das ermittelte thermische Modell kann darüber hinaus verwendet werden, um direkt die Temperatur des zu kalibrierenden Temperaturfühlers zu regeln. Da der Prüfling in der überwiegenden Zahl der Fälle der Temperaturfühler ist, der das trägste thermische Verhalten zeigt, kann mit dieser direkten Regelung des Prüflings die Zeit bis zum Erreichen des thermischen Gleichgewichtes nochmals deutlich reduziert werden.

Keywords: Temperaturkalibrator, Regelung, Thermische Modellierung

\section{Schnelle tragbare Temperaturkalibratoren}

Die meisten in der Industrie und Forschung eingesetzten Temperatursensoren müssen vor ihrem Einsatz kalibriert werden. Dazu sind besonders tragbare Temperiereinrichtungen geeignet, da diese häufig sowohl Heiz- als auch Kühlfunktionen wahrnehmen können [1] und sich diese aufgrund ihrer geringen (thermischen) Masse auch besonders schnell auf die gewünschte Kalibriertemperatur regeln lassen. Dabei lassen sich mit neuen Regelverfahren, unter Beibehaltung der hohen Regelgeschwindigkeit, Regelstabilitäten im sub-Millikelvin-Bereich erreichen [2].

Eine Verkürzung der Kalibrierzeiten, die selbst bei Verwendung eines schnellen tragbaren Kalibrators typischerweise noch in der Größenordnung von 45 Minuten pro Kalibrierpunkt liegen, ist aber dennoch wünschenswert. Einen Ansatzpunkt für solch eine Verkürzung kann man der Richtlinie DKDR 5-1 [3] entnehmen: „Die Kalibrierung eines Thermometers erfolgt nach Erreichen des thermischen Gleichgewichtes sowohl der Temperiereinrichtung als auch des Thermometers selbst." Daraus ergibt sich ein Optimierungspotential, wenn man in einer modellbasierten Regelung das thermische Verhalten des Prüflings mit berücksichtigt.

\section{Thermisches Modell des Prüflings}

Als thermisches Modell zur Berechnung der Temperatur des Prüflings haben wir die folgende Differenzialgleichung (1) mit zunächst unbekannten Zeitkonstanten $\tau_{n}$ verwendet:

$$
\prod_{n=1}^{N}\left[1+\tau_{n} \partial_{t}\right] T_{P}(t)=T_{I n}(t) \text {. }
$$

Dabei ist $T_{P}(t)$ die Temperatur des Prüflings und $T_{I n}(t)$ die zu steuernde Temperatur der internen Referenz im Block (siehe Abb. 1).

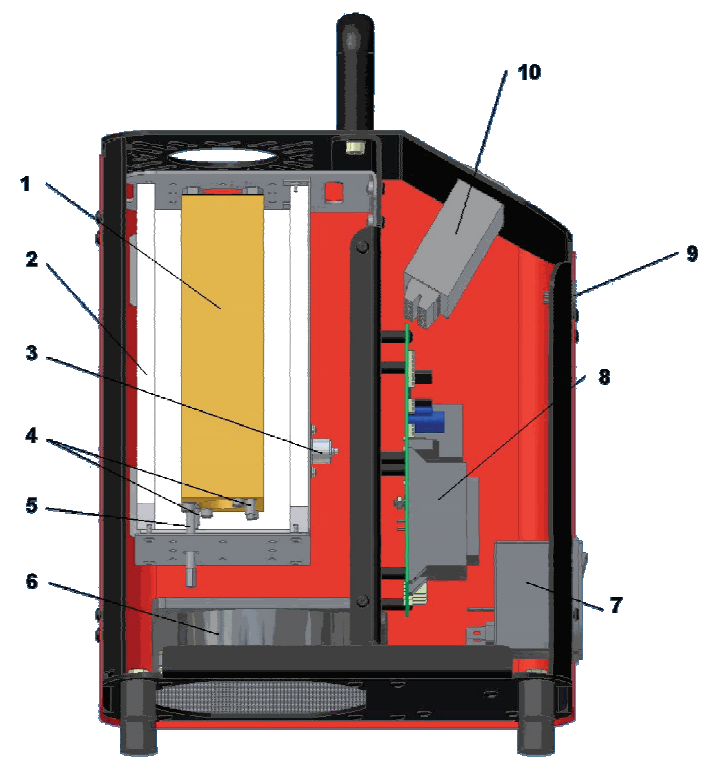

Abb.. 1: Tragbarer Temperaturkalibrator: 1 Block, 2 Isolierung, 3 Temperatursicherung, 4 Heizelemente, 5 Interne Referenz, 6 Lüfter, 7 Netzanschluss, 8 Steuerelektronik, 9 PC Anschluss, 10 Temperaturanzeige. 
Die Zeitkonstanten $\tau_{n}=\frac{1}{\omega_{n}}$ hängen von der verwendeten Kalibratorhülse mit den Bohrungen für den Prüfling und für den optionalen externen Referenzfühler und natürlich vom Prüfling ab. Die Zeitkonstanten können leicht durch Messung der Frequenzantwort der Temperaturanzeige des Prüflings ermittelt werden. Die Frequenzantwort und daraus ermittelte Übertragungsfunktion

$$
G(s)=\frac{\omega_{1} \omega_{2} \omega_{3}}{\left(s+\omega_{1}\right)\left(s+\omega_{2}\right)\left(s+\omega_{3}\right)}
$$

mit den drei Zeitkonstanten $\tau_{n}$ 145s, 4.9s und 3.5s sind in Abb. 2 dargestellt.

\section{Modellbasierte Regelung}

Zunächst haben wir untersucht, wie sich nur unter Verwendung der Temperaturanzeige des internen Referenzsensors als Input für unser thermisches Modell (1), die Temperatur des Prüflings regeln lässt. Dazu wurde ein tragbarer Trockenblockkalibrator des Typs TP3M165E.2, eine Al-Kalibrierhülse mit $60 \mathrm{~mm}$ Durchmesser und ein Prüflingsfühler mit $6 \mathrm{~mm}$ Durchmesser verwendet. Der sich damit ergebende Temperaturverlauf des internen Referenzsensors und der zeitliche Verlauf der Temperaturanzeige des Prüflings sind in den Abbildungen $4 \mathrm{a}$ und $4 \mathrm{~b}$ für eine Regelung von $50^{\circ} \mathrm{C}$ auf $70^{\circ} \mathrm{C}$ und in den Abbildungen $6 a$ und $6 \mathrm{~b}$ für eine Regelung von $70^{\circ} \mathrm{C}$ auf $50^{\circ} \mathrm{C}$ dargestellt. Zum Vergleich sind die entsprechenden zeitlichen Temperaturverläufe für den Fall einer einfachen Regelung des internen Referenzfühlers ohne Berücksichtigung der thermischen Trägheit des Prüflings in den Abbildungen $3 a$ und $3 b$ bzw. $5 \mathrm{a}$ und $5 \mathrm{~b}$ dargestellt. Zunächst ist festzustellen, dass sowohl bei der einfachen Regelung auf den internen Referenzfühler als auch bei der modellbasierten Regelung der interne Referenzfühler die Solltemperatur erreicht und sehr stabil hält. Dies ergibt sich aus der Tatsache, dass das thermische Modell für den Prüfling keinen Offset enthält und somit sichergestellt ist, dass im thermischen Gleichgewicht der interne Referenzfühler die gewünschte Solltemperatur erreicht. Bevor der interne Referenzfühler diesen Sollwert hält macht die Temperatur am Ort des Referenzfühlers aber einen genau kalkulierten und begrenzten „Über- oder Unterschwinger“, der dazu führt, dass der Prüfling statt nach etwa 30 Minuten schon nach etwa 25 Minuten seine Gleichgewichtstemperatur innerhalb des Stabilitätsbandes von +/- 20mK annimmt.

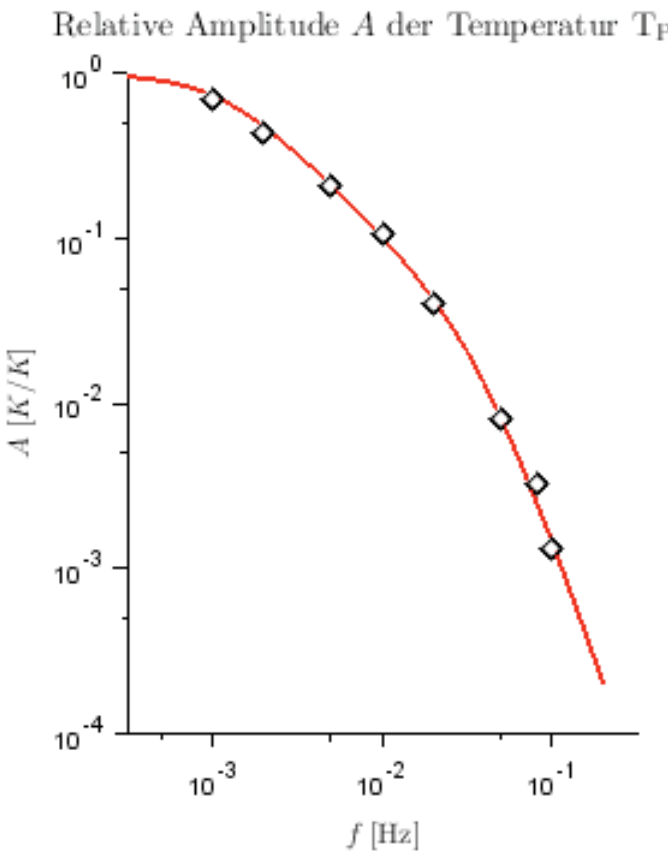

Phase $\phi$ zwischen $\mathrm{T}_{\mathrm{P}}(\mathrm{t})$ und Temperatur $\mathrm{T}_{\mathrm{ln}}(\mathrm{t})$

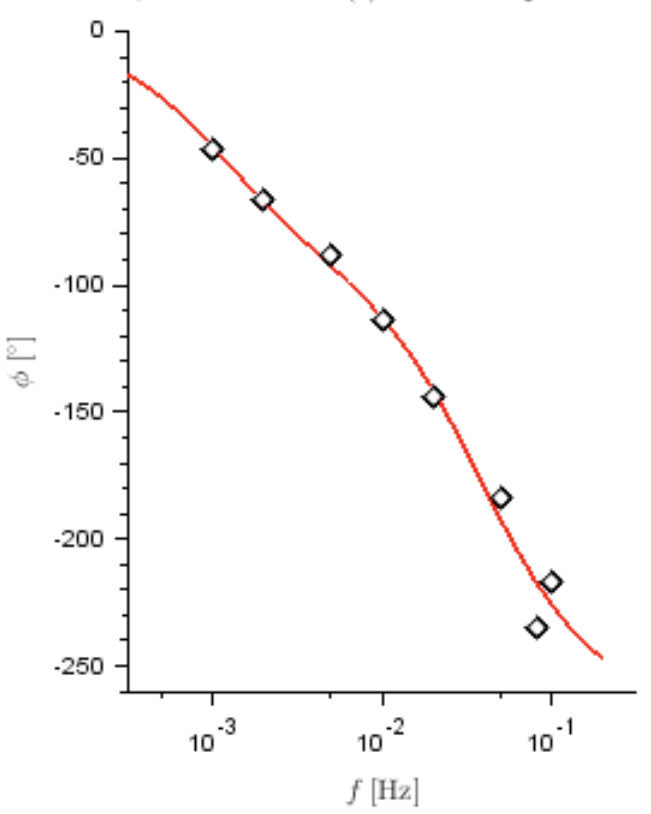

Abb..2: Bode-Diagramm der aus dem thermischen Modell berechneten Übertragungsfunktion $G($ iw) des Prüflings (rote Kurven). Die gemessenen Amplituden und Phasen zwischen der Temperatur des Prüflings und der Temperatur der internen Referenz sind als Rauten dargestellt. 

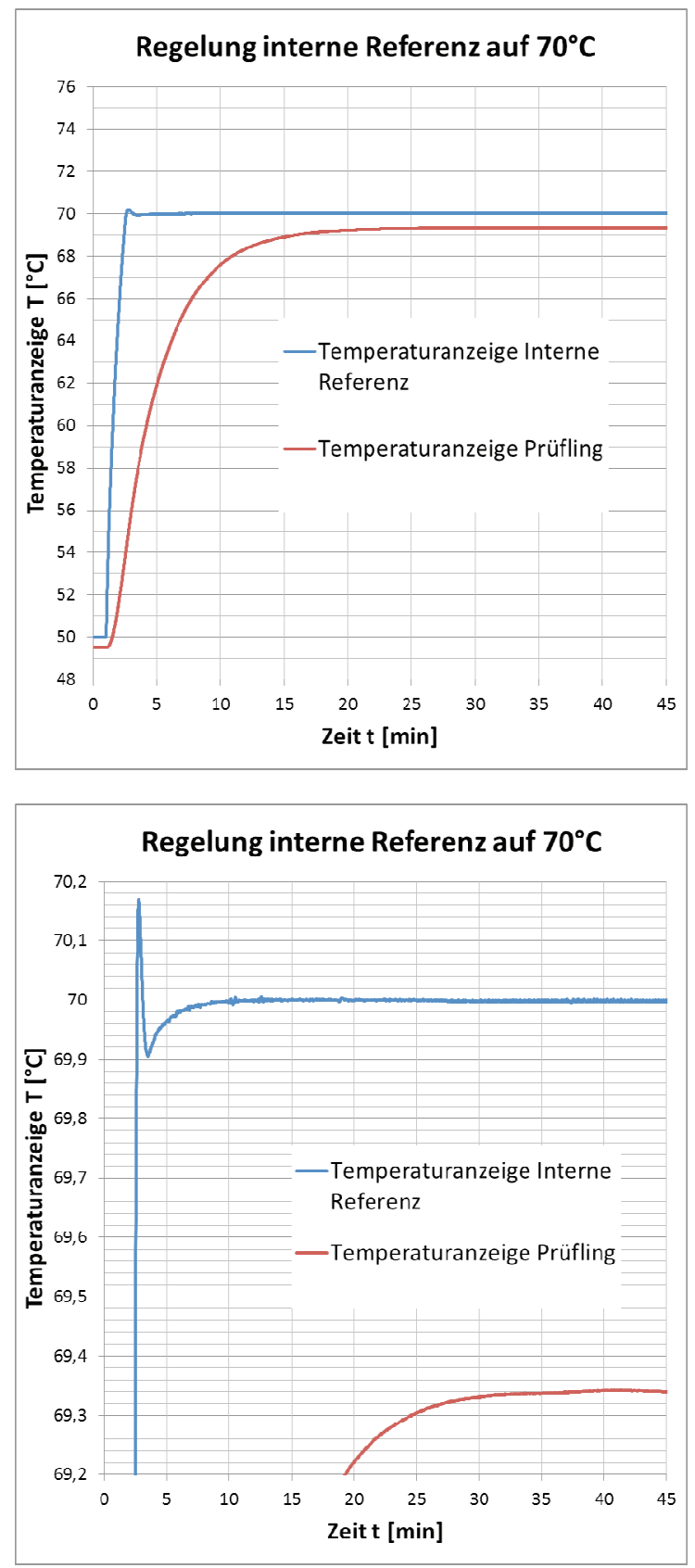

Abb..3: Verlauf der Temperaturanzeigen des internen Referenzfühlers und des Prüflings bei einer Regelung des internen Referenzfühlers auf $70^{\circ} \mathrm{C}$ ohne Berücksichtigung der thermischen Trägheit des Prüflings und der Kalibrierhülse. Vergrößerter Ausschnitt bezüglich des Temperaturbereichs in der unteren Abbildung.
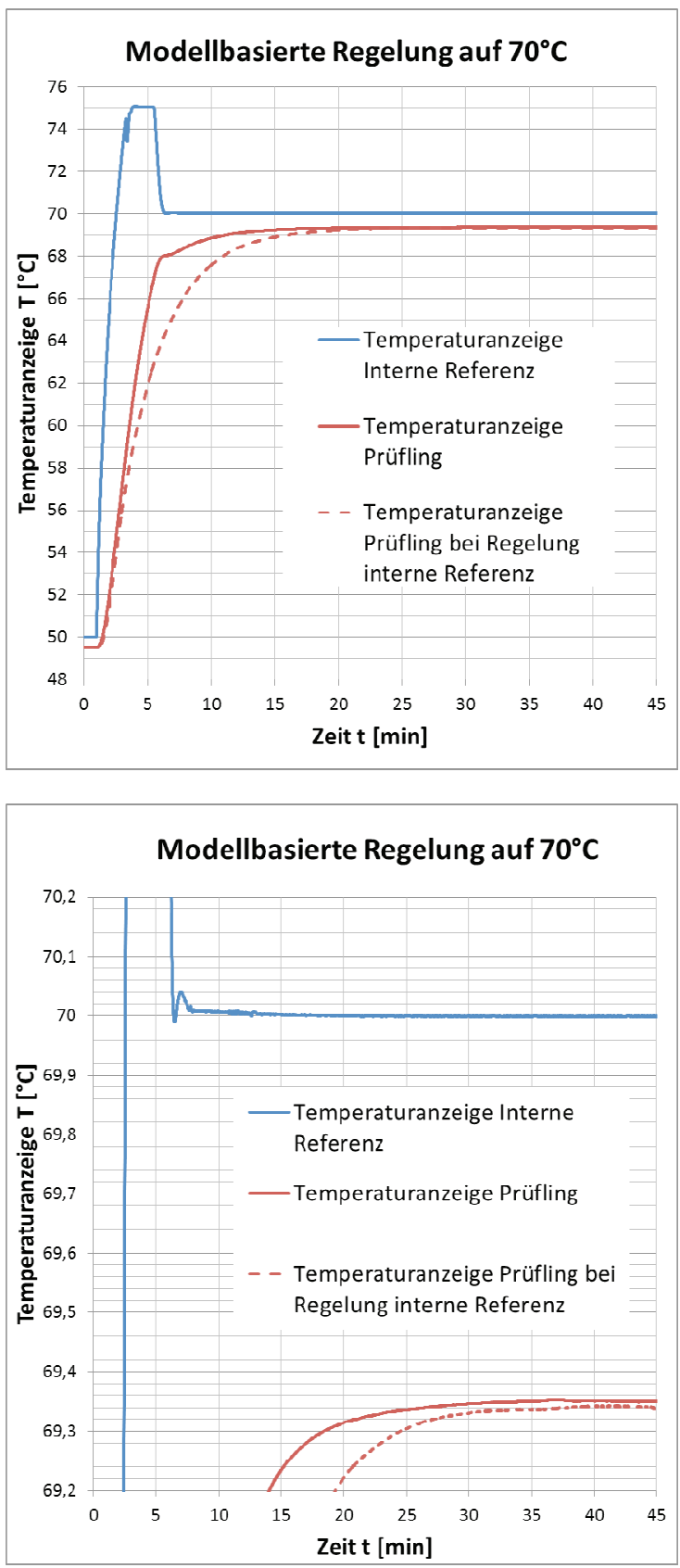

Abb..4: Verlauf der Temperaturanzeigen des internen Referenzfühlers und des Prüflings bei einer Regelung des internen Referenzfühlers auf $70^{\circ} \mathrm{C}$ unter Berücksichtigung der thermischen Trägheit des Prüflings und der Kalibrierhülse durch eine modellbasierte Regelung. Vergrößerter Ausschnitt bezüglich des Temperaturbereichs in der unteren Abbildung. 

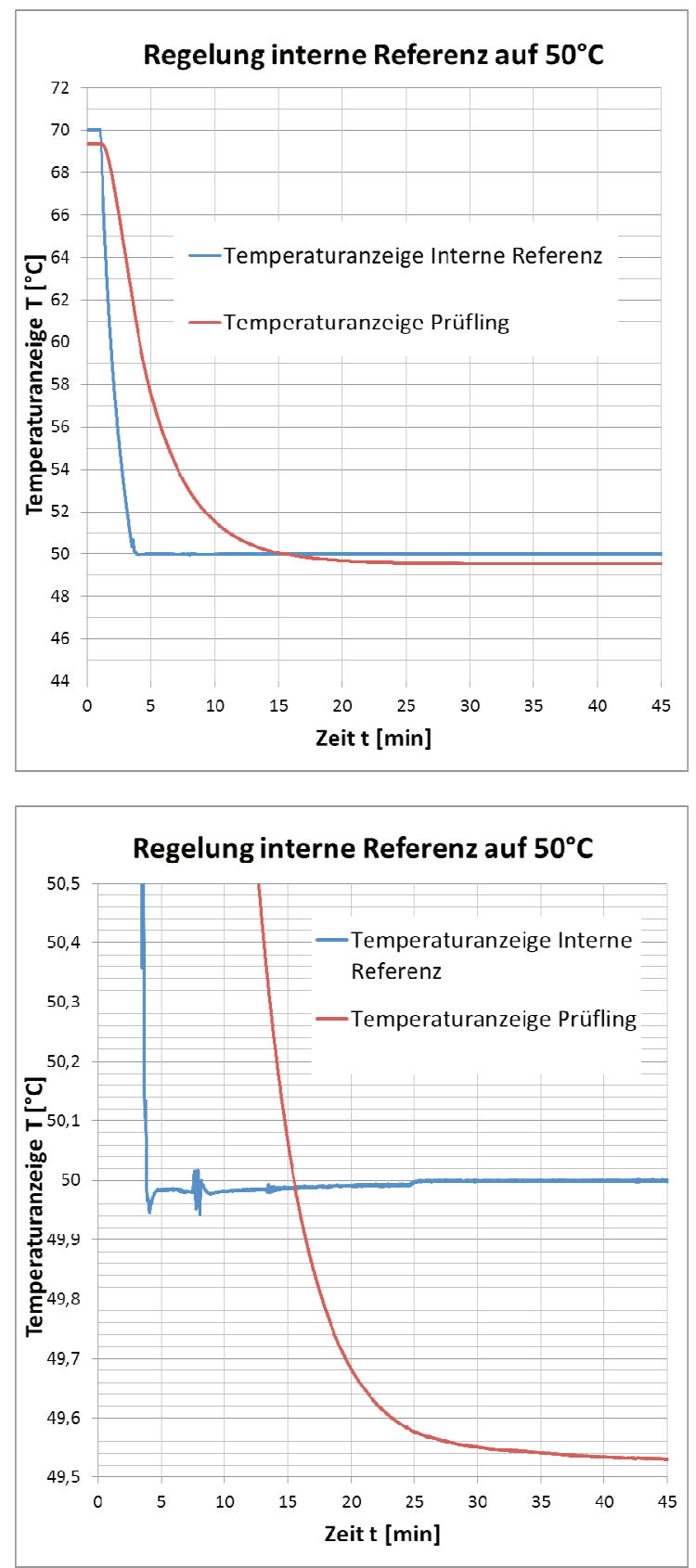

Abb..5: Verlauf der Temperaturanzeigen des internen Referenzfühlers und des Prüflings bei einer Regelung des internen Referenzfühlers auf $50^{\circ} \mathrm{C}$ ohne Berücksichtigung der thermischen Trägheit des Prüflings und der Kalibrierhülse. Vergrößerter Ausschnitt bezüglich des Temperaturbereichs in der unteren Abbildung.
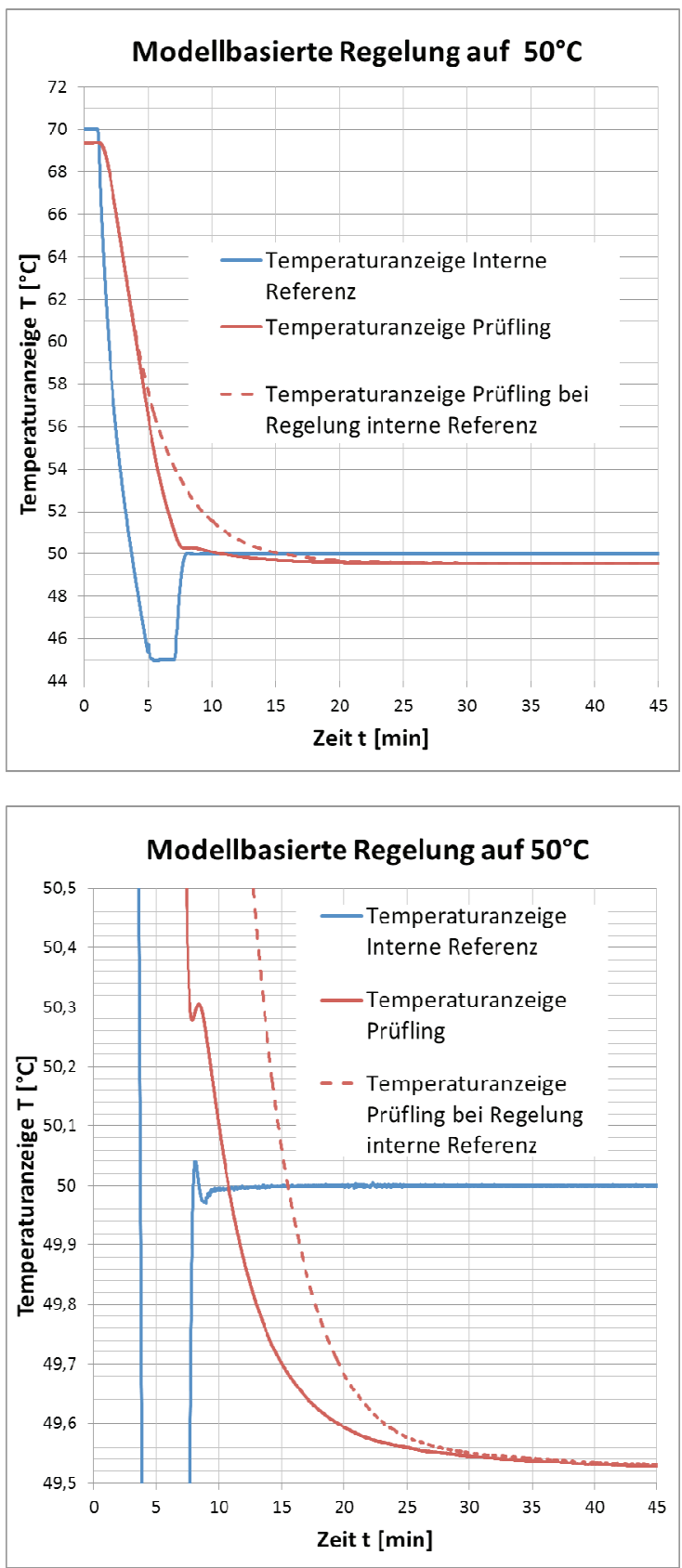

Abb..6: Verlauf der Temperaturanzeigen des internen Referenzfühlers und des Prüflings bei einer Regelung des internen Referenzfühlers auf $50^{\circ} \mathrm{C}$ unter Berücksichtigung der thermischen Trägheit des Prüflings und der Kalibrierhülse durch eine modellbasierte Regelung. Vergrößerter Ausschnitt bezüglich des Temperaturbereichs in der unteren Abbildung. 


\section{Regelung des Prüflings}

In einem zweiten Schritt haben wir untersucht, inwieweit sich die Zeiten bis zum Erreichen des thermischen Gleichgewichtes noch weiter verkürzen lassen, wenn direkt auf die Temperaturanzeige des Prüflings geregelt wird. Dazu haben wir das thermische Modell des Prüflings (1) verwendet, um, wie in [2] und [4] beschrieben, die Temperaturmessdaten des Prüflings mittels eines Kalman-Filters [5] geeignet zu filtern und gleichzeitig die Temperatur des Prüflings in der Kalibrierhülse vorausschauend zu regeln. Der sich daraus ergebende Temperaturverlauf des internen Referenzsensors und der zeitliche Verlauf der Temperaturanzeige des Prüflings sind in den Abbildungen $7 a$ bis $7 c$ für eine Regelung des Prüflings von $50^{\circ} \mathrm{C}$ auf $70^{\circ} \mathrm{C}$ und in den Abbildungen $8 \mathrm{a}$ bis $8 \mathrm{c}$ für eine Regelung des Prüflings von $70^{\circ} \mathrm{C}$ auf $50^{\circ} \mathrm{C}$ dargestellt. In beiden Fällen erreicht der Prüfling äußerst schnell und sogar noch vor der internen Referenz ein thermisches Gleichgewicht. Das heißt, die Wartezeit bis zum Starten einer Kalibrierung ist nun durch die Zeit bestimmt, in der die interne Referenz das gewünschte Stabilitätsband von +/- 20mK erreicht. Dieses ist nach etwa 20 Minuten erreicht.

Für den Fall einer hochgenauen Kalibrierung unter Verwendung eines externen Referenzfühlers in der Kalibrierhülse, reicht es aus zu warten, bis die externe Referenz im thermischen Gleichgewicht mit dem Prüfling ist. Die dagegen deutlich langsamere Thermalisierung des internen Referenzsensors liegt in der schleichenden Temperierung der den Kalibrierblock umgebenden Baugruppen begründet, die eine deutlich schlechtere Wärmeleitfähigkeit als der Metallblock oder die metallische Kalibrierhülse besitzen. Weil der externe Referenzfühler in der Kalibrierhülse typischerweise eine kleinere oder bei gleicher Bauart sogar eine identische thermische Trägheit wie der Prüfling besitzt, sind beide Fühler im thermischen Gleichgewicht, sobald das gewünschte Stabilitätsband von z.B. +/$5 \mathrm{mK}$ durch den Prüfling erreicht wurde. Man kann erkennen, dass dies bei einer Regelung des Prüflings schon nach 15 Minuten der Fall ist.
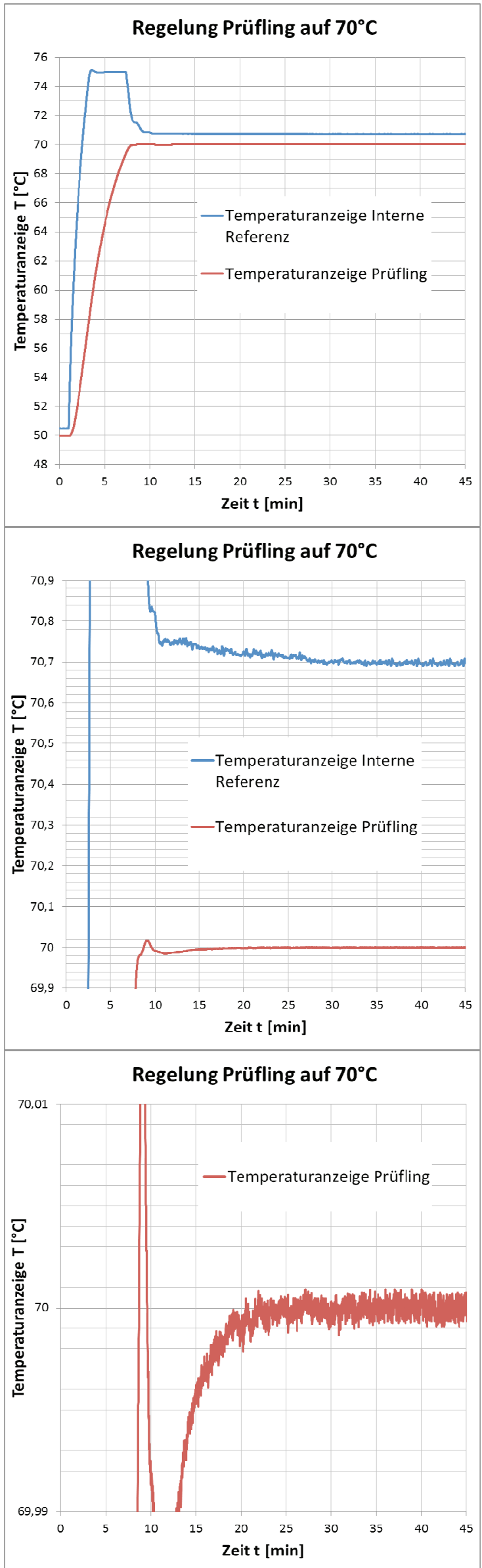

Abb..7: Direkte Regelung des Prüflings auf $70^{\circ} \mathrm{C}$. Darstellungen auf unterschiedlichen Temperaturskalen. 

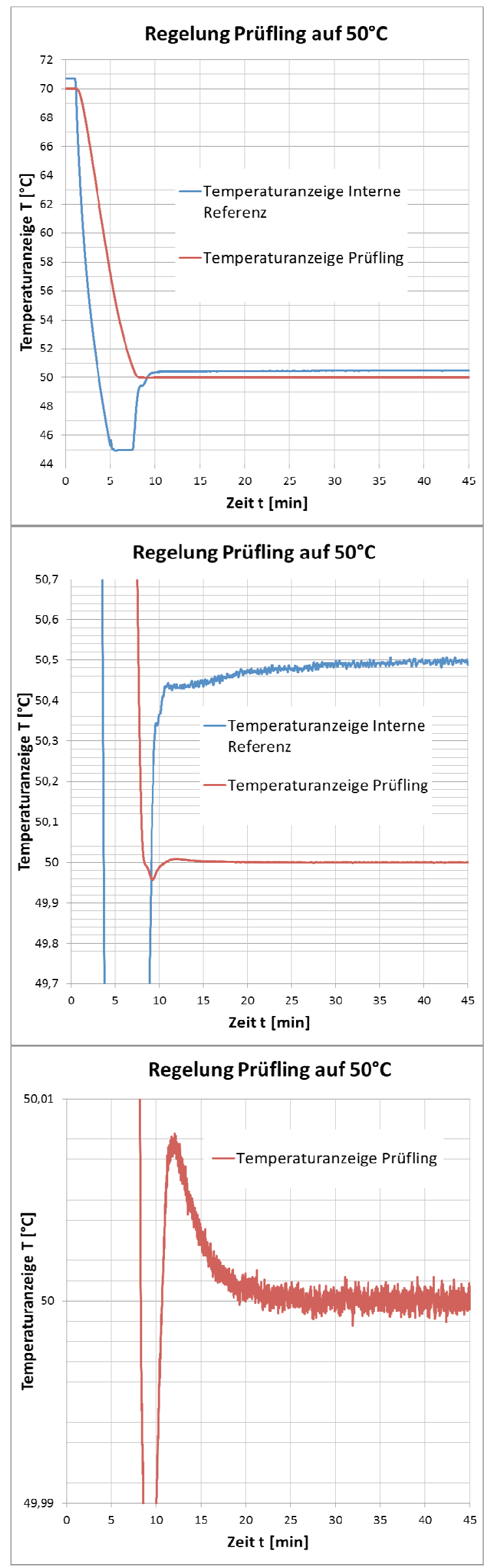

Abb..8: Direkte Regelung des Prüflings auf $50^{\circ} \mathrm{C}$. Darstellungen auf unterschiedlichen Temperaturskalen.

\section{Zusammenfassung}

Durch Berücksichtigung der thermischen Trägheit des Prüflings im Kalibrievolumen im Rahmen einer modellbasierten Regelung oder einer Regelung der Temperaturanzeige des Prüflings lassen sich die Wartezeiten bis zum Erreichen eines ausreichenden thermischen Gleichgewichtes von etwa 30 Minuten auf 25 bis 15 Minuten reduzieren. Bei einer in der industriellen Praxis üblichen Haltezeit der Kalibriertemperatur von 15 Minuten nach Erreichen des thermischen Gleichgewichtes ergibt sich daraus eine mögliche Reduktion der Gesamtkalibrierzeit um bis zu 33\% bzw. eine Steigerung der Anzahl der Kalibriervorgänge in einer festen Betriebszeit um bis zu 50\%.

Bei einer angenommenen Lebensdauer eines tragbaren Temperaturkalibrators von 10000 Betriebsstunden und einer Leistungsaufnahme in der Größenordnung von $1 \mathrm{~kW}$ lässt sich diese Verkürzung der Kalibrierzeiten in eine Energieersparnis von bis zu $5 \mathrm{MWh}$ über die Lebensdauer eines Temperaturkalibrator umrechnen.

Neben den reduzierten Energiekosten und den schnelleren Kalibriervorgängen hat eine Regelung der Temperaturanzeige des Prüflings auch den messtechnischen Vorteil, dass unnötige Kalibrierunsicherheiten aufgrund von Kalibriervorgängen, die vor dem Erreichen eines thermischen Gleichgewichtes gestartet werden, vermieden werden. Damit ist in den nicht seltenen Fällen, in denen dem Ausführenden einer Kalibrierung die Thermalisierungszeit des zu kalibrierenden Temperaturfühlers nicht oder nur ungefähr bekannt ist, eine Regelung der Temperaturanzeige des Prüflings von besonderem Vorteil.

\section{Literaturnachweis}

[1] DE 202005006 710, SIKA Dr. Siebert \& Kühn $\mathrm{GmbH} \&$ Co. KG, "Als Metallblock ausgebildeter Temperaturkalibrator für Thermometer", (2005)

[2] R. Friedrichs, G. Villacrés und E.C. Weiß, „Tragbarer Temperaturkalibrator mit subMillikelvin-Regelstabilität“, Konferenz: Sensoren und Messsysteme 2014, Nürnberg, 17. ITG/GMA-Fachtagung

[3] Richtlinie DKD-R 5-1, "Kalibrierung von Widerstandsthermometern", Ausgabe 10/2003

[4] L. F. Mouzinho, J. V. FonsecaNeto, B. A. Luciano und R. C. S. Freire, „INDIRECT MEASUREMENT OF THE TEMPERATURE VIA KALMAN FILTER“, XVIII IMEKO World Congress, 09/2006

[5] R. E. Kalman, „A New Approach to Linear Filtering and Prediction Problems", Journal of basic Engineering, 82(1), 35-45 (1960) 